\title{
Gauss-Bonnet coupling constant as a free thermodynamical variable and the associated criticality
}

\author{
Wei Xua ${ }^{\mathrm{a}}$, Hao Xu ${ }^{\mathrm{b}}$, Liu Zhao ${ }^{\mathrm{c}}$ \\ School of Physics, Nankai University, Tianjin 300071, People's Republic of China
}

Received: 28 December 2013 / Accepted: 6 July 2014 / Published online: 22 July 2014

(c) The Author(s) 2014. This article is published with open access at Springerlink.com

\begin{abstract}
The thermodynamic phase space of GaussBonnet (GB) AdS black holes is extended, taking the inverse of the GB coupling constant as a new thermodynamic pressure $P_{\mathrm{GB}}$. We studied the critical behavior associated with $P_{\mathrm{GB}}$ in the extended thermodynamic phase space at fixed cosmological constant and electric charge. The result shows that when the black holes are neutral, the associated critical points can only exist in five dimensional GB-AdS black holes with spherical topology, and the corresponding critical exponents are identical to those for the Van der Waals system. For charged GB-AdS black holes, it is shown that there can be only one critical point in five dimensions (for black holes with either spherical or hyperbolic topologies), which also requires the electric charge to be bounded within some appropriate range; while in $d>5$ dimensions, there can be up to two different critical points at the same electric charge, and the phase transition can occur only at temperatures which are not in between the two critical values.
\end{abstract}

\section{Introduction}

Thermodynamic properties of black holes have been studied for many years, especially in anti-de Sitter (AdS) spacetime due to the AdS/CFT correspondence [1-4]. An outstanding feature for AdS black holes is the so-called HawkingPage phase transition, which can happen between stable large black holes and thermal gas in AdS spacetime [5]. Thermodynamics of charged black holes in AdS spacetime has also been intensively studied. The asymptotically AdS charged black holes admit a gauge duality description via a dual thermal field theory. This dual description suggests that charged AdS black holes exhibit a critical behavior in the $Q-\phi$ diagram which is reminiscent of the liquid-gas phase transition

\footnotetext{
a e-mail: xuweifuture@mail.nankai.edu.cn

b e-mail: physicshx@gmail.com

ce-mail: 1zhao@nankai.edu.cn
}

in a Van der Waals system [6-13], where $Q$ and $\phi$ denote the electric charge and potential, respectively.

Recently, this picture has been substantially extended. The idea of including the variation of the cosmological constant $\Lambda$ in the first law of black hole thermodynamics has acquired increasing attention [14-21]. By studying the critical behaviors of AdS black holes in the extended phase space, i.e. the $P-V$ diagram [22-35], where $P$ is the thermodynamic pressure associated with the cosmological constant, which takes the value

$P=-\frac{1}{8 \pi} \Lambda=\frac{(d-1)(d-2)}{16 \pi \ell^{2}}$

in the geometric units $G_{N}=\hbar=c=k=1$, with $\ell$ being the $d$-dimensional AdS radius, $V$ is the conjugate "thermodynamic volume" $[17,20,36,37]$, the analogy in the $Q-\phi$ diagram of AdS charged black hole with a Van der Waals system has been further enhanced. Both systems share the critical exponents and have extremely similar phase diagrams. This analogy has been generalized to higher dimensional charged black holes [23,26,30], rotating black holes [33-35], GaussBonnet (GB) black holes [13,22], $f(R)$ black hole [28], black holes with scalar hair [29,32], black holes with nonlinear source [27], and Born-Infeld black holes [24] in AdS space.

The inclusion of $P-V$ variables in the thermodynamics of AdS black holes is not just an artificial game to play. There exist several reasons why $\Lambda$ should be included as a thermodynamical variable [23]. One may suppose that there is some more fundamental theory in which some physical constants such as Yukawa and gauge coupling constants, Newton's constant, or cosmological constant, may not be fixed values but may be dynamical ones arising from the vacuum expectation values and hence can vary. Thus, it is natural to add variations of these "constants" into the first law of black hole thermodynamics [38,39]. Besides, the cosmological constant term is necessary in the first law of black hole thermodynamics, in order to get a consistent Smarr relation for black hole thermodynamics from the scaling argument 
[15]. A similar situation appears for the Born-Infeld black holes [40-42] and GB black holes [22]. To get a consistent Smarr relation by scaling arguments, one has to introduce the Born-Infeld and GB parameter terms into the first law of black holes. Once one takes the cosmological constant as the thermodynamic pressure in the first law, the black hole mass $M$ should be explained as enthalpy rather than internal energy of the system [15].

In this paper, we will revisit the first law of thermodynamics for GB black holes with emphasis on the role of GB coupling $\alpha$. It is well known that GB gravity theory has two AdS solutions with effective cosmological constants [43-47]

$\Lambda_{\mathrm{eff}}=-\frac{(d-1)(d-2)}{4 \tilde{\alpha}}\left(1-\delta \sqrt{1+8 \frac{\tilde{\alpha} \Lambda}{(d-1)(d-2)}}\right)$,

where $\tilde{\alpha}=(d-3)(d-4) \alpha . \delta=+1$ corresponds to the general relativistic (GR) branch and has a GR limit as $\alpha \rightarrow 0$, while $\delta=-1$ corresponds to the GB branch and does not have the GR limit. It has been shown by Boulware and Deser that the GB branch is unstable and the graviton is a ghost, while the GR branch is stable and is free from ghosts [43]. Thus we shall only consider the GR branch with $\delta=+1$. By a simple analogy with the previously mentioned $P-V$ criticality for AdS black holes, one may tend to consider $P_{\text {eff }} \propto-\Lambda_{\text {eff }}$ as a thermodynamic pressure. However, for two reasons, we will not take this point of view. Firstly, $\Lambda_{\text {eff }}$ is a complicated combination of two parameters $\Lambda$ and $\alpha$ and we wish to understand the role of each parameter independently. Secondly, in the enthalpy description of the first law, $P_{\text {eff }}$ always appear in differential form, i.e. in the term $V_{\text {eff }} \mathrm{d} P_{\text {eff }}$, and it is reasonable to decompose $\mathrm{d} P_{\text {eff }}$ as a combination of $\mathrm{d} \Lambda$ and $\mathrm{d} \alpha$, thanks to (2). Moreover, we have a good reason to consider

$P_{\mathrm{GB}} \equiv \frac{1}{8 \pi \alpha}$

instead of $\alpha$ as a component of the thermodynamic pressure: it is $\frac{1}{\alpha}$ which scales like a pressure. This is where our considerations depart from the previous work [22], which took $\alpha$ and its conjugate $\mathcal{A}$ as a pair of thermodynamic quantities. This consideration is also different from the geometric formulation for Lovelock theories [48], which contain GaussBonnet gravity as a special case. Nonetheless, it will become clear that using $P_{\mathrm{GB}}$ and its conjugate as a pair of thermodynamic variables indeed reveals novel criticalities of the theory, which are otherwise difficult to describe in terms of $\alpha-\mathcal{A}$ variables.

We will consider the criticality associated with the new thermodynamic variables $P_{\mathrm{GB}}$ and its conjugate $V_{\mathrm{GB}}$ in the extended phase space of charged GB-AdS black holes. It will be shown that this kind of critical behavior is different from the previously mentioned $P-V$ criticality, and that the spatial curvature of the AdS black hole horizon plays an important role in such criticalities.

The paper is organized as follows. Section 2 is devoted to the thermodynamics of GB-AdS black holes in the extended phase space with $P_{\mathrm{GB}}$ and $V_{\mathrm{GB}}$. In Sect. 3, we consider the criticality associated with $P_{\mathrm{GB}}$ for static neutral GB-AdS black holes. The case for static charged GB-AdS black holes is discussed in Sect. 4. Finally, some concluding remarks are given in the last section.

\section{Extended thermodynamics of GB-AdS black holes}

The action of $d$-dimensional Einstein-GB-Maxwell theory with a bare cosmological constant $\Lambda$ reads

$$
\begin{array}{r}
I=\frac{1}{16 \pi} \int \mathrm{d}^{d} x \sqrt{-g}\left[R-2 \Lambda+\alpha\left(R_{\mu \nu \gamma \delta} R^{\mu \nu \gamma \delta}\right.\right. \\
\left.\left.-4 R_{\mu \nu} R^{\mu \nu}+R^{2}\right)\right]-\frac{1}{4} \int \mathrm{d}^{d} x \sqrt{-g} F_{\mu \nu} F^{\mu \nu},
\end{array}
$$

where the GB coupling $\alpha$ has dimension [length] ${ }^{2}$ and can be identified with the inverse string tension with positive value [43] if the theory is incorporated in string theory, thus we shall consider only the case $\alpha>0$. Of course, we take the spacetime dimension $d \geq 5$, since in $d=4$ dimensions, the integration of the GB density $\mathcal{L}_{\mathrm{GB}}=R_{\mu \nu \gamma \delta} R^{\mu \nu \gamma \delta}-$ $4 R_{\mu \nu} R^{\mu \nu}+R^{2}$ is a topological number and has no dynamics.

The $d$-dimensional static charged GB-AdS black hole solution arising from the field equations associated with the action (4) is well known to take the form

$\mathrm{d} s^{2}=-f(r) \mathrm{d} t^{2}+\frac{1}{f(r)} \mathrm{d} r^{2}+\mathrm{d} \Omega_{d-2, k}^{2}$,

which should be accompanied by a standard Coulomb potential for the Maxwell field, where $\mathrm{d} \Omega_{d-2, k}^{2}$ represents the line element of a $(d-2)$-dimensional maximally symmetric Einstein space with constant curvature $(d-2)(d-3) k$, where $k=1,0$ and -1 correspond to the spherical, Ricci flat and hyperbolic topology of the black hole horizons, respectively. The metric function $f(r)$ is given by $[43,44,49,50]$

$$
\begin{aligned}
& f(r)=k+\frac{r^{2}}{2 \tilde{\alpha}} \\
& \times\left(1-\sqrt{1-\frac{4 \tilde{\alpha}}{\ell^{2}}+\frac{64 \pi \tilde{\alpha} M}{(d-2) r^{d-1}}-\frac{2 \tilde{\alpha} Q^{2}}{(d-2)(d-3) r^{2 d-4}}}\right),
\end{aligned}
$$

where $M$ and $Q$ are the mass and charge (in geometric units) of the black hole, respectively.

Since we are going to discuss the thermodynamics of the black hole in the extended phase space by introducing extra thermodynamical variables $P-V$ and $P_{\mathrm{GB}}-V_{\mathrm{GB}}$, the black 
hole mass $M$ should be identified with the enthalpy $H \equiv M$ rather than the internal energy of the gravitational system [15]. It follows from (6) that $H$ can be expressed in terms of the horizon radius $r_{+}$

$$
\begin{aligned}
H=\left[\frac{(d-2) r_{+}^{d-3}}{16 \pi}\left(k+\frac{r_{+}^{2}}{\ell^{2}}+\frac{k^{2}}{8 \pi r_{+}^{2} P_{\mathrm{GB}}}\right)\right. \\
\left.+\frac{Q^{2}}{32 \pi(d-3) r_{+}^{d-3}}\right],
\end{aligned}
$$

where $r_{+}$is the largest root of $f(r)$. The Hawking temperature of the black hole is given by

$$
\begin{aligned}
T & =\frac{1}{4 \pi} f^{\prime}\left(r_{+}\right) \\
& =\frac{\frac{(d-1) r_{+}^{4}}{\ell^{2}}+(d-3) k r_{+}^{2}+\frac{(d-5) k^{2}}{8 \pi P_{\mathrm{GB}}}-\frac{Q^{2}}{2(d-2) r_{+}^{2 d-8}}}{4 \pi r_{+}\left(r_{+}^{2}+\frac{k}{4 \pi P_{\mathrm{GB}}}\right)} .
\end{aligned}
$$

Other thermodynamic quantities are well known in the literature $[44,50]$. For example, the entropy $S$ and electric potential $\Phi$ are given by

$$
\begin{aligned}
& S=\frac{r_{+}^{d-2}}{4}\left(1+\frac{(d-2) k}{4 \pi(d-4) r_{+}^{2} P_{\mathrm{GB}}}\right), \\
& \Phi=\frac{Q}{8 \pi(d-3) r_{+}^{d-3}} .
\end{aligned}
$$

Note that here we have treated the cosmological constant and GB coupling constant as free thermodynamical variables, and their conjugate quantity thermodynamic volumes $V$ and $V_{\mathrm{GB}}$ are given, respectively, by

$$
\begin{aligned}
& V=\left(\frac{\partial H}{\partial P}\right)_{S, Q, P_{\mathrm{GB}}}=\frac{r_{+}^{d-1}}{d-1}, \\
& V_{\mathrm{GB}} \equiv\left(\frac{\partial H}{\partial P_{\mathrm{GB}}}\right)_{S, Q, P}=-\frac{(d-2) k^{2}}{128 \pi^{2} P_{\mathrm{GB}}^{2}} r_{+}^{d-5} .
\end{aligned}
$$

Note that these thermodynamic volumes are in no way linked to the geometric volume of the black hole: in fact, from the point of view of static observers located outside of the black hole, there is no such notion of a geometric volume of the black hole at all. The physical meaning of these thermodynamic volumes remains obscure. Therefore, the negative value of $V_{\mathrm{GB}}$ should not be considered unacceptable. Anyway, the negativity of $V_{\mathrm{GB}}$ does not prevent us from studying criticality in the extended thermodynamic phase space, since in the following we shall take $r_{+}$- which is always positiveinstead of $V_{\mathrm{GB}}$ as an equation of state (EOS) parameter associated with $P_{\mathrm{GB}}$.

An important relation in black hole thermodynamics is the Smarr relation. In order to obtain the correct Smarr relation for the above-mentioned charged static GB-AdS black hole, we will now make some scaling arguments. Since the black hole enthalpy $H$ (i.e. the mass $M$ ) is a homogeneous function of entropy $S$, electric charge $Q$, and thermodynamic pressures $P$ and $P_{\mathrm{GB}}$, and as $M$ scales as [length] ${ }^{d-3}, S$ scales as [length] ${ }^{d-2}, Q$ scales as [length] ${ }^{d-3}, P$ and $P_{\mathrm{GB}}$ scales as [length $]^{-2}$ (see, e.g. $[15,48]$ ), we find that the Smarr relation for the black hole under consideration reads

$$
(d-3) H=(d-2) T S+(d-3) Q \Phi-2 P V-2 P_{\mathrm{GB}} V_{\mathrm{GB}} \text {. }
$$

This is certainly different from the Smarr relation known from the previous literature, because we have now extended the thermodynamic phase space and have taken $P_{\mathrm{GB}}$ and $V_{\mathrm{GB}}$ as a new pair of conjugate thermodynamic variables. Correspondingly, the first law of black hole thermodynamics is generalized as

$\mathrm{d} H=T \mathrm{~d} S+\Phi \mathrm{d} Q+V \mathrm{~d} P+V_{\mathrm{GB}} \mathrm{d} P_{\mathrm{GB}}$.

In the rest of this paper, we shall consider criticality associated with the new variable $P_{\mathrm{GB}}$, taking $P$ and $Q$ as fixed parameters. In order to study the criticality of black holes, it is necessary to work with the Gibbs free energy $G$, which can be obtained via the Legendre transformation,

$$
\begin{aligned}
G= & G\left(T, Q, P, P_{\mathrm{GB}}\right)=H-T S \\
= & \frac{r_{+}^{d-3}(d-2)}{16 \pi}\left(k+\frac{r_{+}^{2}}{\ell^{2}}\right) \\
& -\frac{T r_{+}^{d-2}}{4}\left(1+\frac{(d-2) k}{4 \pi P_{\mathrm{GB}}(d-4) r_{+}^{2}}\right) \\
& +\frac{k^{2}(d-2) r_{+}^{d-5}}{128 \pi^{2} P_{\mathrm{GB}}}+\frac{Q^{2} r_{+}^{3-d}}{32 \pi(d-3)} .
\end{aligned}
$$

We shall also make use of the internal energy

$$
\begin{aligned}
U & =H-P_{\mathrm{GB}} V_{\mathrm{GB}} \\
& =\frac{(d-2) r_{+}^{d-3}}{16 \pi}\left(k+\frac{r_{+}^{2}}{\ell^{2}}+\frac{k^{2}}{4 \pi r_{+}^{2} P_{\mathrm{GB}}}\right)+\frac{Q^{2}}{32 \pi(d-3) r_{+}^{d-3}},
\end{aligned}
$$

while evaluating the heat capacity at fixed $r_{+}$, where $P_{\mathrm{GB}}$ is to be taken as a function of the temperature $T$, thanks to the EOS

$$
P_{\mathrm{GB}}=\frac{\left((5-d) k+8 T \pi r_{+}\right)(d-2) k}{8 \pi\left[\left(\frac{r_{+}^{2}(d-1)}{\ell^{2}}+(d-3) k-4 T \pi r_{+}\right)(d-2) r_{+}^{2}-\frac{Q^{2}}{2 r_{+}^{2 d-8}}\right]},
$$

which arises from (8). Note that for $k=0, P_{\mathrm{GB}}$ is identically zero, thus $P_{\mathrm{GB}}$ and $V_{\mathrm{GB}}$ lose their role as a pair of thermodynamic variables. So, in this paper, we shall always assume $k \neq 0$. This EOS is quite different from the Van der Waals equation,

$$
P=\frac{T}{v-b}-\frac{a}{v^{2}},
$$


which states that the pressure $P$ is a linear function of the temperature $T$, if the specific volume $v$ is kept fixed. Nonetheless, the usual method for studying criticalities for the Van der Waals system still works, as will be seen below.

Before proceeding, let us note that there are some natural constraints on the allowed range of $P_{\mathrm{GB}}$ : Firstly, a welldefined vacuum solution with $M=0, Q=0$ results in [22] $P_{\mathrm{GB}} \geq \frac{1}{2 \pi \ell^{2}}$ (i.e. the dimensionless pressure $\frac{P_{\mathrm{GB}}}{P}$ has a lower bound: $\left.\frac{P_{\mathrm{GB}}}{P} \geq \frac{8}{(d-1)(d-2)}\right)$; Secondly, the non-negative definiteness of the black hole entropy (9) gives another constraint [51]: $P_{\mathrm{GB}} \geq \frac{d-2}{4(d-4) \pi r_{+}^{2}}$ for $k=-1$.

\section{Criticality for static neutral GB-AdS black holes associated with $\boldsymbol{P}_{\mathrm{GB}}$}

\subsection{Critical behavior in five and six dimensions}

For simplicity, we begin our study with the case of neutral black holes. The black hole enthalpy (7) is reduced to

$H=\frac{(d-2) r_{+}^{d-3}}{16 \pi}\left(k+\frac{r_{+}^{2}}{\ell^{2}}+\frac{k^{2}}{8 \pi P_{\mathrm{GB}} r_{+}^{2}}\right)$,

and the Gibbs free energy becomes

$$
\begin{aligned}
G= & \frac{(d-2) r_{+}^{d-3}}{16 \pi}\left(k+\frac{r_{+}^{2}}{\ell^{2}}+\frac{k^{2}}{8 \pi P_{\mathrm{GB}} r_{+}^{2}}\right) \\
& -\frac{T r_{+}^{d-2}}{4}\left(1+\frac{(d-2) k}{4 \pi(d-4) r_{+}^{2} P_{\mathrm{GB}}}\right) .
\end{aligned}
$$

The EOS of the black holes simplifies into

$$
P_{\mathrm{GB}}=\frac{\left((5-d) k+8 T \pi r_{+}\right) k}{8 \pi\left(\frac{r_{+}^{2}(d-1)}{\ell^{2}}+(d-3) k-4 T \pi r_{+}\right) r_{+}^{2}} .
$$

The critical point is determined as the inflection point on the $P_{\mathrm{GB}}-r_{+}$diagram, i.e.,

$$
\left.\frac{\partial P_{\mathrm{GB}}}{\partial r_{+}}\right|_{r_{+}=r_{c}, T=T_{c}}=\left.\frac{\partial^{2} P_{\mathrm{GB}}}{\partial r_{+}^{2}}\right|_{r_{+}=r_{c}, T=T_{c}}=0,
$$

and $\left.\frac{\partial^{2} P_{\mathrm{GB}}}{\partial r_{+}^{2}}\right|_{r_{+}=r_{c}+0^{+}, T=T_{c}}$ and $\left.\frac{\partial^{2} P_{\mathrm{GB}}}{\partial r_{+}^{2}}\right|_{r_{+}=r_{c}+0^{-}, T=T_{c}}$ should have different signs, where we have used the subscript $c$ to stand for the quantities at the critical point. Using (21), the two conditions in (22) becomes a pair of very complicated algebraic equations for $r_{c}$ and $T_{c}$, which, of course, depend on the spacetime dimension $d$ and the signature of the spatial curvature $k$ of the black hole horizons. For $k=-1$, the pair of equations arising from (22) can never have a solution with real and positive $r_{c}$. Therefore, we are left with only the choice $k=+1$. In this case, eliminating $T_{c}$ from the above pair of equations, we get a single simplified equation determining the critical radius $r_{c}$,

$36(d-1)^{2} R_{c}^{2}-12(d-1)(2 d-9) R_{c}+(d-3)(7 d-39)=0$,

where we have introduced

$R_{c}=\frac{r_{c}^{2}}{\ell^{2}}$.

The solutions to this equation read

$R_{c}=\frac{2 d-9 \pm \sqrt{-3(d-2)(d-6)}}{6(d-1)}$.

In order to find a real positive $R_{c}$, we need to take $d=5$ or $d=6$. For $d=5$, only the + branch of the solution is allowed, and we can check that around the corresponding solution $r_{c}, \frac{\partial^{2} P_{\mathrm{GB}}}{\partial r_{+}^{2}}$ indeed changes signature, thus the solution is indeed a critical point. For $d=6$, the two branches of solutions (25) degenerate, and $\frac{\partial^{2} P_{\mathrm{GB}}}{\partial r_{+}^{2}}$ does not change its signature around the corresponding $r_{c}$, so we conclude that there is no critical point in six dimensions.

\section{(1) Critical point in five dimensions}

In five dimensions, we have

$R_{c}=\frac{1}{6}, \quad r_{c}=\frac{\sqrt{6}}{6} \ell, \quad P_{\mathrm{GB}}^{c}=\frac{9}{2 \pi \ell^{2}}, \quad T_{c}=\frac{\sqrt{6}}{2 \pi \ell}$,

from which we can easily find

$\frac{P_{\mathrm{GB}}^{c} r_{c}}{T_{c}}=\frac{3}{2}$.

This relation is universal in the sense that it is independent of all parameters. This result is very similar to the one in the Van der Waals system, which has $\frac{P_{c} r_{c}}{T_{c}}=\frac{3}{8}$. This makes it more conceivable to use the horizon radius $r_{+}$instead of the thermodynamic volume $V_{\mathrm{GB}}$ as an EOS parameter, since $V_{\mathrm{GB}}$ does not lead to such a parameter independent relation. Since we are taking $P$ (i.e. $\ell$ via (1)) as a constant parameter, it is better to work in units of $P$ or $\ell$ and re-express the critical parameters in dimensionless form,

$\frac{r_{c}}{\ell}=\frac{\sqrt{6}}{6}, \quad \frac{P_{\mathrm{GB}}^{c}}{P}=6$.

In five dimensions, the previously mentioned lower bound for the dimensionless pressure is $\frac{P_{\mathrm{GB}}}{P} \geq \frac{2}{3}$, and the above critical value is well above this lower bound. So, the criticality can always be found in this case. We can have a clear look at this in the $P_{\mathrm{GB}}-r_{+}$diagrams and $G-T$ diagrams which are presented in Fig. 1.

It can be seen from Fig. 1 that only for the isotherms with $T>T_{c}$ there exist a local minimum and a maximum. Along the segment of the isotherm between these two extrema, we 


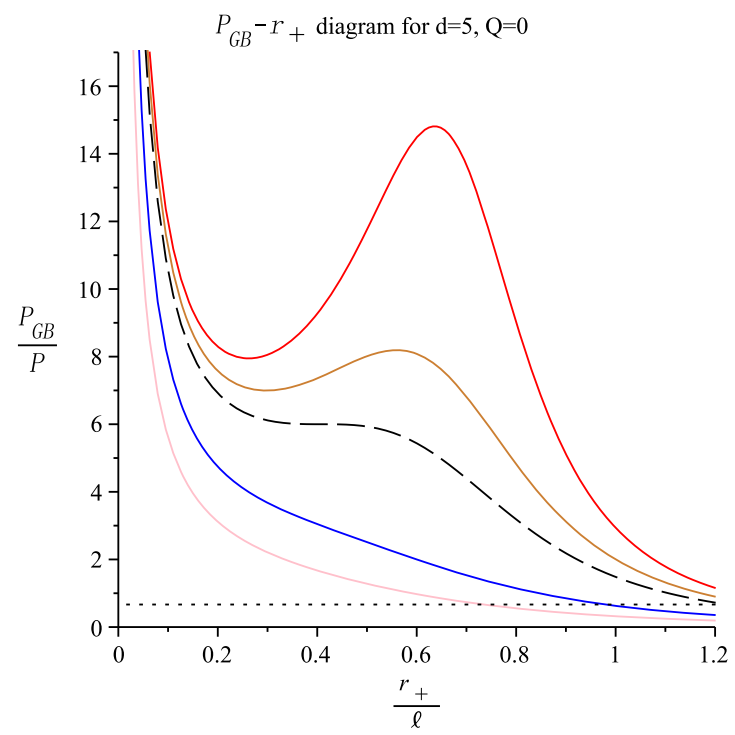

Fig. 1 The isotherm $\left(P_{\mathrm{GB}}-r_{+}\right.$plots at arbitrary constant $\ell$ on the left $)$ and Gibbs free energy at fixed $P_{\mathrm{GB}}(G-T$ plots at $\ell=\sqrt{10}$ on the right) for five dimensional static neutral GB-AdS black holes with $k=1$. In both plots, the dotted line corresponds to that for the lower bound for $P_{\mathrm{GB}}$. The temperatures of the isotherms decrease from top to bottom. The lower two isotherms contain no phase transition. The

have $\frac{\partial P}{\partial r_{+}}>0$, which implies that the black holes is in a thermally unstable phase. For the $\frac{\partial P}{\partial r_{+}}<0$ regions, the black holes are thermally stable, corresponding, respectively, to a small and a large black hole at the same temperature. Physically speaking, the system at $T>T_{c}$ is in thermal equilibrium between the stable small black hole and large black hole phases, because the unstable, medium sized black hole phase cannot physically exist. The true isotherm for such cases should be replaced by a steeply descending segment and a slowly descending segment joined by a straight, horizontal (i.e. an isobar $P_{\mathrm{GB}}=P^{*}=$ const.) segment which can be determined by Maxwell's equal area law. When the critical temperature $T_{c}$ is reached, the shape of the isotherm will undergo a significant change. The two extrema merge into a single inflection point, and we can no longer distinguish between the stable small and large black holes. The isotherms with $T<T_{C}$ no longer contain any extrema and there is only one branch with positive compression coefficient corresponding to thermally stable black holes.

Turning to the Gibbs free energy plots, we see that each of the curves corresponding to $P>P_{\mathrm{GB}}^{c}$ can extend to $T>T_{c}$ and contains a "swallow tail" segment, which is a typical feature in first order phase transitions. From $P=P_{c}$ and downwards, the "swallow tail" disappears, with $P=P_{c}$ corresponding to the critical point.

\section{(2) No critical point in six dimensions}

In six dimensions, we can obtain the following solution:

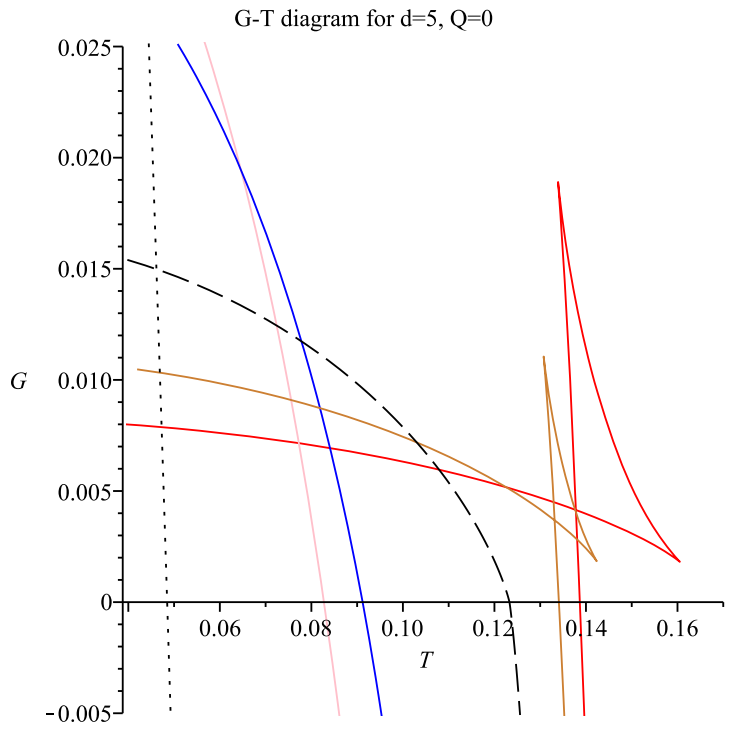

critical isotherm $T=T_{c}$ is depicted in a dashed line, while the upper two isotherms correspond to two-phase equilibrium states. The pressure $P_{\mathrm{GB}}$ on the right plots increases from left to right, and the "swallow tail" behavior appears only when $P_{\mathrm{GB}}>P_{\mathrm{GB}}^{c}$, which corresponds to a first order phase transition

$$
R_{c}=\frac{1}{10}, \quad r_{c}=\frac{\sqrt{10}}{10} \ell, P_{\mathrm{GB}}^{c}=\frac{5}{2 \pi \ell^{2}}, T_{c}=\frac{\sqrt{10}}{2 \pi \ell}
$$

for the equations given in (22). However, it is easy to check that $\frac{\partial^{2} P_{\mathrm{GB}}}{\partial r_{+}^{2}}$ does not change its signature around the above $r_{c}$; therefore, the above solution corresponds actually to an extremum, rather than an inflection point on an isotherm. Consequently, each isotherm contains one and only one extremum (which is a maximum). This means that at each temperature, the value of $P_{\mathrm{GB}}$ has an upper bound, black holes with $P_{\mathrm{GB}}$ bigger than the upper bound simply could not exist. For smaller values of $P_{\mathrm{GB}}$, there are two different black holes at each temperature: a small unstable black hole and a large stable black hole. The small black hole phase cannot physically persist because of its thermal instability, and there is no phase equilibrium in this case.

In Fig. 2, we present the $P_{\mathrm{GB}}-r_{+}$and $G-T$ diagrams in six dimensions. The dimensionless pressure and radius is used in the $P_{\mathrm{GB}}-r_{+}$plots. The dashed curves corresponding to the special values given in (29) play no particular role as compared to the other curves. It can be seen on the $G-T$ plots that for each $P_{\mathrm{GB}}$, the $G(T)$ curve is "<"-shaped, with the lower branch corresponding to the stable large black hole phase.

\subsection{Critical exponents in five dimensions}

In this subsection, we will study the scaling behaviors of some physical quantities near the criticality and compute the corresponding critical exponents in five dimensions. The 


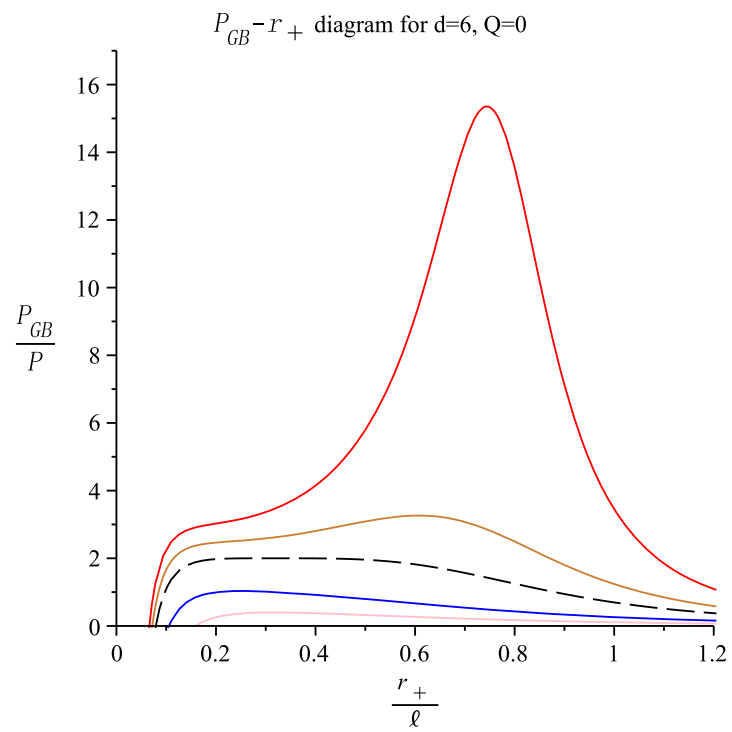

Fig. 2 The isotherm $\left(P_{\mathrm{GB}}-r_{+}\right.$plots at arbitrary constant $\ell$ on the left $)$ and Gibbs free energy at fixed $P_{\mathrm{GB}}(G-T$ plots at $\ell=\sqrt{10}$ on the right $)$ for six dimensional static neutral GB-AdS black holes with $k=1$. The

construction is analogous to the scaling behavior of the Van de Waals liquid-gas system, so we begin by reviewing the scaling laws for the Van de Waals system. Near the critical point, the critical behavior of a Van der Waals liquid-gas system can be characterized by the following critical exponents [52]:

$C_{v} \sim\left(\frac{\left|T-T_{c}\right|}{T_{c}}\right)^{-\alpha}$,

$\frac{v_{g}-v_{l}}{v_{c}} \sim\left(-\frac{T-T_{c}}{T_{c}}\right)^{\beta}$,

$\kappa_{T} \sim\left(\frac{\left|T-T_{c}\right|}{T_{c}}\right)^{-\gamma}$,

$P-P_{c} \sim\left(v-v_{c}\right)^{\delta}$,

where $C_{v}=\left.T\left(\frac{\partial S}{\partial T}\right)\right|_{v}$ is the heat capacity at constant volume, $\kappa_{T}=-\left.v^{-1}\left(\frac{\partial v}{\partial P}\right)\right|_{T}$ is the isothermal compressibility, the subscripts $g$ and $l$ stand for quantities in the gaseous and liquid phases, respectively. The critical exponents take the following values:

$\alpha=0, \quad \beta=1 / 2, \quad \gamma=1, \quad \delta=3$.

In our case, we need to replace the specific volume $v$ by the black hole radius $r_{+}$and study the corresponding scaling properties. The liquid and gaseous phases for the Van der Waals system should also be replaced by the small and large black hole phases, respectively. Since we shall be interested in the properties near criticality, we introduce the following dimensionless parameters, which tend to zero near the critical point:

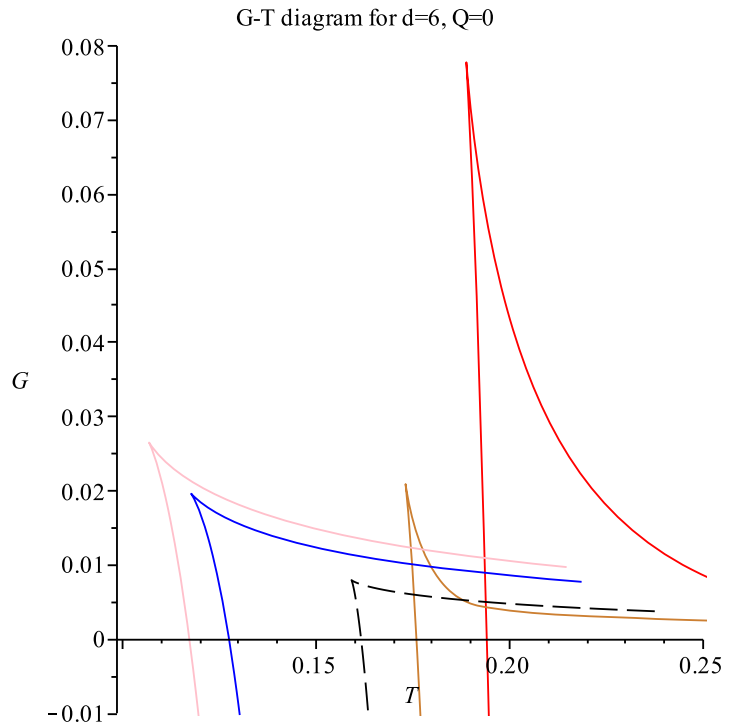

temperature of the isotherms decrease from top to bottom. There is no "swallow tail" behavior in the $G-T$ diagram showing that there is no phase equilibrium

$t=\frac{T}{T_{c}}-1, \quad \phi=\frac{r_{+}}{r_{c}}-1 \quad p=\frac{P_{\mathrm{GB}}}{P_{\mathrm{GB}}^{c}}-1$.

Using these new parameters and inserting the critical values (26) into the $\mathrm{EOS}$ (21) at $d=5, k=+1$, we get the following dimensionless EOS:

$p+1=\frac{t+1}{(\phi+1)\left(-3 \phi t-\phi-3 t+1+\phi^{2}\right)}$.

The Taylor series expansion for (36) at the critical point gives

$p=4 t+6 t \phi-\phi^{3}+O\left(t \phi^{2}, \phi^{4}\right)$,

where we have neglected terms of order $\phi^{4}$ and $t \phi^{2}$ or higher as did in [23]. It will be shown below that $t$ and $\phi^{2}$ are of the same order.

Using Maxwell's equal area law, we obtain the following equation:

$0=\int_{\phi_{l}}^{\phi_{s}} \phi \frac{\mathrm{d} p}{\mathrm{~d} \phi} \mathrm{d} \phi \Rightarrow-\frac{3}{2}\left(\phi_{l}^{4}-\phi_{s}^{4}\right)+6 t\left(\phi_{l}^{2}-\phi_{s}^{2}\right)=0$,

where the subscripts $s$ and $l$ stand for small and large stable black hole phases, respectively. On the other hand, the physical phase equilibrium condition (i.e. the isobar pressure condition) gives

$\left.p\right|_{\phi_{s}}=\left.p\right|_{\phi_{l}} \Rightarrow 6 t\left(\phi_{l}-\phi_{s}\right)-\left(\phi_{l}^{3}-\phi_{s}^{3}\right)=0$.

Equations (38) and (39) together give a unique non-trivial solution $\left(\phi_{s} \neq \phi_{l}\right)$ :

$\phi_{s}=-\sqrt{6 t}, \quad \phi_{l}=\sqrt{6 t}$.

We conclude that the coexistence of small and large black hole phases requires $t>0$. In other words, only when $T>$ 
$T_{c}$ can the two stable black hole phases exist at the same pressure. This is different from a Van der Waals liquid-gas system where $T<T_{c}$ is required for a phase equilibrium. Equation (40) can be rewritten as

$r_{+l}-r_{+s} \propto\left(T-T_{c}\right)^{1 / 2}$.

This scaling behavior is in analogy to (31), which gives us the critical exponent $\beta=\frac{1}{2}$.

The isothermal radial compressibility can be calculated as follows:

$\kappa_{T} \equiv-\left.\frac{1}{r_{+}} \frac{\partial r_{+}}{\partial P_{\mathrm{GB}}}\right|_{r_{+}=r_{c}} \propto-\left.\frac{1}{\frac{\partial p}{\partial \phi}}\right|_{\phi=0}=-\frac{1}{6 t}$.

This implies that

$\kappa_{T} \propto-\left(T-T_{C}\right)^{-1}$,

which gives the critical exponent $\gamma=1$. In addition, it can easily be seen that $\left.p\right|_{t=0}=-\phi^{3}$, i.e.

$P_{\mathrm{GB}}-P_{\mathrm{GB}}^{c} \propto-\left(r_{+}-r_{c}\right)^{3}$,

which tells us $\delta=3$.

To evaluate the heat capacity near the critical point, we need to substitute $d=5, k=+1$ into (16) and then calculate

$\left.C_{r_{+} \rightarrow r_{c}} \equiv \frac{\partial U}{\partial T}\right|_{r_{+} \rightarrow r_{c}}=-\frac{\sqrt{6} \ell}{48 \pi^{2} T^{2}}$.

This result is completely regular in $T$, showing that the critical exponent $\alpha=0$. We see that though the EOS of our system is quite different from that of the Van der Waals system, the resulting set of critical exponents are exactly the same as that for the Van der Waals system. It is not a surprise that these critical exponents satisfy the following thermodynamic scaling laws:

$\alpha+2 \beta+\gamma=2, \quad \alpha+\beta(1+\delta)=2$,

$\gamma(1+\delta)=(2-\alpha)(\delta-1), \quad \gamma=\beta(\delta-1)$.

\section{Criticality for static charged GB-AdS black holes}

\subsection{Critical behavior in five dimensions}

Having now understood the critical behavior of the static neutral GB-AdS black holes associated with the new thermodynamic variables $P_{\mathrm{GB}}$ and $r_{+}$, we turn our attention to the static charged GB-AdS black holes. The analysis will be basically parallel to the neutral cases, though the details are more complicated due to the presence of extra parameters.

When $d=5$, the numerator of the EOS (17) can be simplified. Therefore we will discuss the two cases $d=5$ and $d>5$ separately. First consider the case $d=5$. The EOS (17) reduces to

$$
P_{\mathrm{GB}}=\frac{3 k T r_{+}}{6 r_{+}^{2}\left(2 \frac{r_{+}^{2}}{\ell^{2}}+k-2 T \pi r_{+}\right)-\frac{Q^{2}}{2 r_{+}^{2}}} .
$$

This simplified EOS allows us to study the critical behavior analytically. Inserting (46) into (22), one finds that the critical horizon radius has to satisfy the following equation:

$24 R_{c}^{3}-4 k R_{c}^{2}-\frac{5 Q^{2}}{\ell^{4}}=0, \quad R_{c} \equiv \frac{r_{c}^{2}}{\ell^{2}}$.

This equation has three analytical roots

$$
\begin{aligned}
R_{c 1}= & \frac{1}{\ell^{2}}\left(\frac{1}{36} x_{c}^{1 / 3}+\frac{1}{9} \frac{k^{2} \ell^{4}}{x_{c}^{1 / 3}}+\frac{1}{18} k \ell^{2}\right), \\
R_{c 2}= & \frac{1}{\ell^{2}}\left(-\frac{1}{2}\left[\frac{1}{36} x_{c}^{1 / 3}+\frac{1}{9} \frac{k^{2} \ell^{4}}{x_{c}^{1 / 3}}\right]\right. \\
& \left.+i \frac{\sqrt{3}}{2}\left[\frac{1}{36} x_{c}^{1 / 3}-\frac{1}{9} \frac{k^{2} \ell^{4}}{x_{c}^{1 / 3}}\right]+\frac{1}{18} k \ell^{2}\right), \\
R_{c 3}= & \frac{1}{\ell^{2}}\left(-\frac{1}{2}\left[\frac{1}{36} x_{c}^{1 / 3}+\frac{1}{9} \frac{k^{2} \ell^{4}}{x_{c}^{1 / 3}}\right]\right. \\
& \left.-i \frac{\sqrt{3}}{2}\left[\frac{1}{36} x_{c}^{1 / 3}-\frac{1}{9} \frac{k^{2} \ell^{4}}{x_{c}^{1 / 3}}\right]+\frac{1}{18} k \ell^{2}\right),
\end{aligned}
$$

where

$x_{c}=4860 Q^{2} \ell^{2}+8 k^{3} \ell^{6}+36 \sqrt{15} \sqrt{1215 Q^{2}+4 k^{3} \ell^{4}} Q \ell^{2}$.

Not all of these roots are real and positive, and we need to choose the real positive root by some physical arguments.

Now assume that $R_{C}$ is given. Then the other critical quantities can be evaluated with ease,

$$
\begin{aligned}
& T_{c}=\frac{3 Q^{2}+4 k r_{c}^{4}}{8 r_{c}^{5} \pi}=\frac{k+9 R_{c}}{5 \pi \ell \sqrt{R_{c}}}, \\
& P_{\mathrm{GB}}^{c}=\left.P_{\mathrm{GB}}\right|_{r_{+}=r_{c}, T=T_{c}}=\frac{3}{20 \pi} \frac{k+9 R_{c}}{\ell^{2} k R_{c}\left(k-3 R_{c}\right)}, \\
& \frac{P_{\mathrm{GB}}^{c} r_{c}}{T_{c}}=\frac{3 k}{4\left(k-3 R_{c}\right)} .
\end{aligned}
$$

Unfortunately, the right hand side of (52) does not look so neat as in (27), because it depends on the physical parameter $R_{c}$, or alternatively, on $Q$ and $\ell$, thanks to the relation (47). The critical temperature $T_{c}$ and pressure $P_{\mathrm{GB}}^{c}$ must be positive. This leads to

$k+9 R_{c}>0$,

$k\left(k-3 R_{c}\right)>0$.

For $k=1, R_{c}$ must lie in the range $0<R_{c}<\frac{1}{3}$; for $k=-1$, $R_{c}$ must obey $R_{c}>\frac{1}{9}$. 


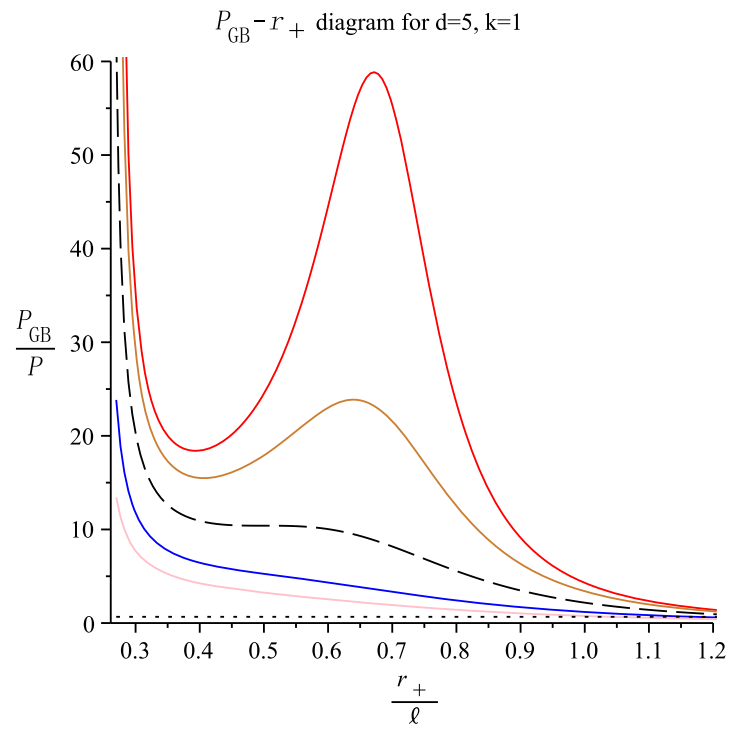

Fig. 3 The $P_{\mathrm{GB}}-r_{+}$(at arbitrary constant $\ell$ on the left) and $G-T$ (at $\ell=8$ on the right) diagrams of five dimensional static charged GB-AdS black holes at $k=1$ and $Q=\frac{\sqrt{10}}{20} \ell^{2}$. On the left plots, the temperature of isotherms decrease from top to bottom, with the dashed line being the

On the other hand, the squared charge $Q^{2}$ can be taken as a function of $R_{C}$ at the critical point due to (47),

$Q^{2}\left(R_{c}\right)=\frac{4 R_{c}^{2} \ell^{4}\left(6 R_{c}-k\right)}{5}$.

For $k=+1$, positivity of the squared charge gives a tighter bound for $R_{c}$, i.e. $\frac{1}{6}<R_{c}<\frac{1}{3}$. For $k=-1$, the bound on $R_{c}$ does not get tighter; however, $Q^{2}$ is still bounded: $Q>\frac{2 \sqrt{3}}{27} \ell^{2}$. Summarizing the above discussions, we have

1. for $k=1$ (spherical horizon), the critical horizon radius and charge need be in this region:

$$
\frac{1}{6} \leq R_{c}<\frac{1}{3}, \quad 0 \leq Q<\frac{2 \sqrt{5}}{15} \ell^{2}
$$

2. for $k=-1$ (hyperbolic horizon), the bounds are given as follows:

$$
R_{c}>\frac{1}{9}, \quad Q>\frac{2 \sqrt{3}}{27} \ell^{2}
$$

In both cases we have $1215 Q^{2}+4 k^{3} \ell^{4}>0$. This in turn implies that only the root (48) of (47) is real positive, and we always have only one critical point in five dimensions.

In Fig. 3, we depict the $P_{\mathrm{GB}}-r_{+}$(at arbitrary constant $\ell$ ) and $G-T$ curves (at $\ell=8$ ) for the special choice $k=+1$, and $Q=\frac{\sqrt{10}}{20} \ell^{2}$. One finds that the "swallow tail" behavior appears only when $P>P_{c}, T>T_{c}$, which corresponds to a phase transition. Such a phase transition is first order for

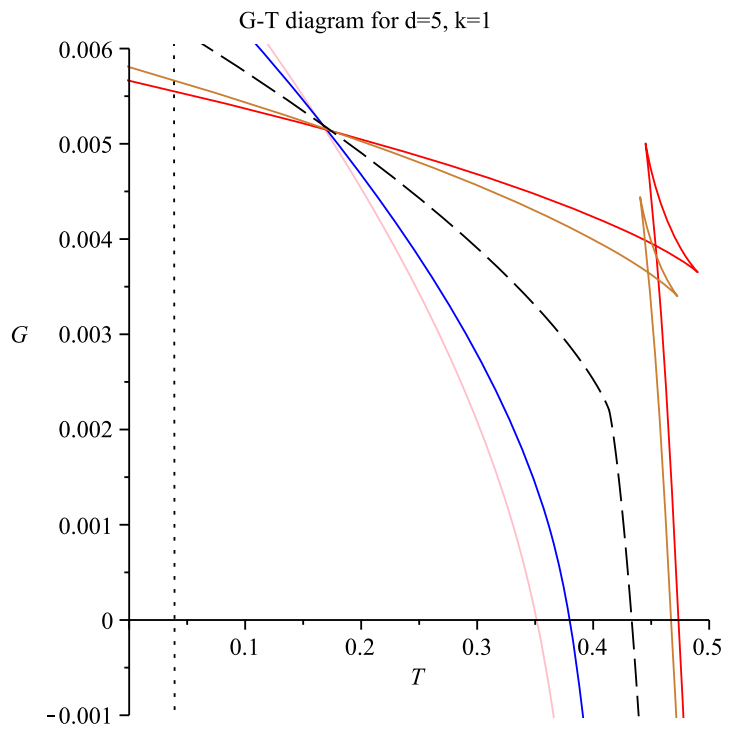

isotherm at the critical temperature and the dotted line corresponding to that for the lower bound for $P_{\mathrm{GB}}$. On the right plots, the "swallow tail" behavior appears only when $P>P_{c}$, which correspond to the phase transition

$T>T_{c}$, while it becomes second order at $T=T_{c}$ just as in the case of the Van der Waals system. In this case, the phase transition can always be found, as the pressure at the phase transition point is much bigger than the lower bound for the pressure, as shown in Fig. 3. The critical behavior is very similar to the case of neutral black holes. We can even show that the critical exponents are kept unchanged in the presence of electric charge, as will be shown for generic values of $Q$.

The dimensionless EOS in terms of the parameters defined in (35) is very complicated in the presence of an electric charge $Q$. However, its Taylor series expansion is simple enough and can be expressed as follows:

$p=a_{10} t+a_{11} t \phi+a_{03} \phi^{3}+O\left(t \phi^{2}, \phi^{4}\right)$,

where

$a_{10}=\frac{4\left(2 k+3 R_{c}\right)}{5\left(k-3 R_{c}\right)}, a_{11}=\frac{6\left(k+9 R_{c}\right)}{5\left(k-3 R_{c}\right)}, a_{03}=\frac{2\left(k-9 R_{c}\right)}{k-3 R_{c}}$.

To calculate the critical exponents, we shall follow the same procedure as had led to (40). It follows that

$\phi_{s}=-\sqrt{-\frac{a_{11}}{a_{03}} t}=-\sqrt{-\frac{3\left(k+9 R_{c}\right)}{5\left(k-9 R_{c}\right)}} t$,

$\phi_{l}=\sqrt{-\frac{a_{11}}{a_{03}} t}=\sqrt{-\frac{3\left(k+9 R_{c}\right)}{5\left(k-9 R_{c}\right)} t}$.

For all allowed values of $R_{c}$ and $Q$ satisfying the bound (56) or (57), we have always $\frac{a_{11}}{a_{03}}=\frac{3\left(k+9 R_{c}\right)}{5\left(k-9 R_{c}\right)}<0$, therefore the 
coexistence of the small and large black hole phases requires $t>0$, i.e. the phase transition appears only at temperature higher than $T_{c}$. The relation (41) still holds and the critical exponent $\beta=\frac{1}{2}$.

The isothermal compressibility can be calculated easily, giving rise to $\kappa_{T} \propto-\frac{1}{a_{11} t}$, which indicates that the critical exponent $\gamma=1$. In addition, it can be shown that $\left.p\right|_{t=0}=a_{03} \phi^{3}$, which gives the critical exponent $\delta=3$. Finally, $C_{r_{+} \rightarrow r_{c}}=-\frac{k r_{c}\left(3 r_{c}^{3}+2 k \ell^{2}\right)}{20 \pi^{2} \ell^{2} T^{2}}$ has no singular behavior at the critical points, and so $\alpha=0$. This completes the proof that the critical exponents are kept unchanged even in the presence of an electric charge in five dimensions.

\subsection{Critical behavior in $d>5$ dimensions}

The critical behavior in $d>5$ dimensions is more difficult to analyze, not only because of the more complicated EOS, but also because the conditions (22) for the critical points become a much more complicated set of algebraic equations, making it harder to solve analytically. To understand such a criticality, we shall proceed in two different ways: For generic spacetime dimension $d>5$, we shall take some particular choice for the electric charge $Q$, which allows us to obtain the critical point parameters analytically. On the other hand, in six dimensions, we shall study the isotherms and $G-T$ curves numerically, taking $k=+1$ and $Q=0.01 \ell^{3}$ at arbitrary constant $\ell$. This allows us to understand the phase structure in this particular dimension.

\subsubsection{Analytical critical point in generic dimensions $d>5$}

In generic dimensions $d>5$, the critical point conditions (22) become a set of very complicated algebraic equations in $T_{c}$ and $r_{c}$. To gain some insights into the solutions, we take the following particular choice for the electric charge $Q$ :

$Q=\sqrt{\varrho} r_{c}^{d-3}$,

or, in terms of the dimensionless parameter $R_{c}=\frac{r_{c}^{2}}{\ell^{2}}$,

$Q=\sqrt{\varrho} R_{c}^{(d-3) / 2} \ell^{d-3}$,

where $\varrho$ is a dimensionless parameter because $Q$ has the dimension [length] ${ }^{d-3}$.

With the aid of the computer algebra system Maple, we can eliminate $T_{c}$ from the pair of equations that follow from (22), which yields a single algebraic equation for $R_{c}$,

$$
\begin{aligned}
& 72(d-1)^{2}(d-2)^{2} R_{c}{ }^{2} \\
& \quad-(6(d-1)(d-2)(5 d-14)(2 d-7) \varrho \\
& \left.\quad+24 k(d-1)(2 d-9)(d-2)^{2}\right) R_{c} \\
& \quad+(2 d-5)(d-4)(2 d-7)^{2} \varrho^{2}
\end{aligned}
$$

$$
\begin{aligned}
& +k(d-2)\left(14 d^{3}-177 d^{2}+697 d-858\right) \varrho \\
& +2 k^{2}(d-3)(7 d-39)(d-2)^{2}=0 .
\end{aligned}
$$

The particular choice (62) for the electric charge makes the resulting equation (63) of second order in $R_{c}$, which is exactly solvable. The corresponding $T_{c}$ given by

$$
\begin{aligned}
T_{c}= & \mathcal{N}^{-1} k(d-5)(\varrho d+2 k d-4 \varrho-6 k) \\
& \times\left(k d^{2}-4 \varrho d^{2}+18 R_{c} d^{2}+13 k d+24 \varrho d\right. \\
& \left.-54 R_{c} d-30 k-41 \varrho+36 R_{c}\right),
\end{aligned}
$$

where

$$
\begin{aligned}
\mathcal{N}=( & 52 k^{2} d^{3}+10 k \varrho d^{3}-8 \varrho^{2} d^{3}-356 k^{2} d^{2} \\
& -51 k \varrho d^{2}+44 \varrho^{2} d^{2}+576 k^{2} d+24 k R_{c} d^{3} \\
& -360 k R_{c} d^{2}+912 k R_{c} d+23 k \varrho d+12 \varrho d^{3} R_{c} \\
& -30 \varrho d^{2} R_{c}+6 \varrho d R_{c}-46 \varrho^{2} d \\
& -144 k^{2}+198 k \varrho-576 k R_{c}-35 \varrho^{2} \\
& \left.+12 \varrho R_{c}\right) \pi \ell \sqrt{R_{c}} .
\end{aligned}
$$

For $d>5$, there can be up to two different critical points of critical radius $r_{c}=\ell \sqrt{R_{c}}$ for a given $\varrho$ with appropriate value. However, please bear in mind that these two critical points do not correspond to the same electric charge, because the charge $Q$ and $R_{c}$ are related via (62). Moreover, in the expression (64) for $T_{c}$, there is a factor $(d-5)$, which implies that both solutions to (63) correspond to $T_{c}=0$ when $d=$ 5. In fact, the five dimensional critical point with $T_{c} \neq 0$ described in the previous subsection does not correspond to any of the solutions of (63) with $d=5$.

The explicit solutions to (63) read

$$
\begin{aligned}
R_{c \pm}= & \frac{1}{6(d-1)}\left[(2 d-9) k+\frac{1}{4}(2 d-7)(5 d-14) \varrho \pm \sqrt{3 \Delta}\right] \\
\Delta= & 3(2 d-7)^{2} \varrho^{2}+8 k(2 d-1)(d-4) \varrho \\
& -16 k^{2}(d-2)(d-6) .
\end{aligned}
$$

We can check that for $d>5, \frac{\partial^{2} P_{\mathrm{GB}}}{\partial r_{+}^{2}}$ changes signature around both solutions $r_{c \pm}$; thus the solutions indeed correspond to critical points. Using this solution, all other critical parameters can be evaluated with ease, though the concrete results are too complicated to be reproduced here. Please note that the real positivity of $R_{c \pm}$ and the corresponding $T_{c \pm}$ and $P_{\mathrm{GB}}^{c \pm}$ naturally impose some bounds on $\varrho$, so the two critical points will appear only for electric charges within some specific region, just like in the previously studied five dimensional case. 


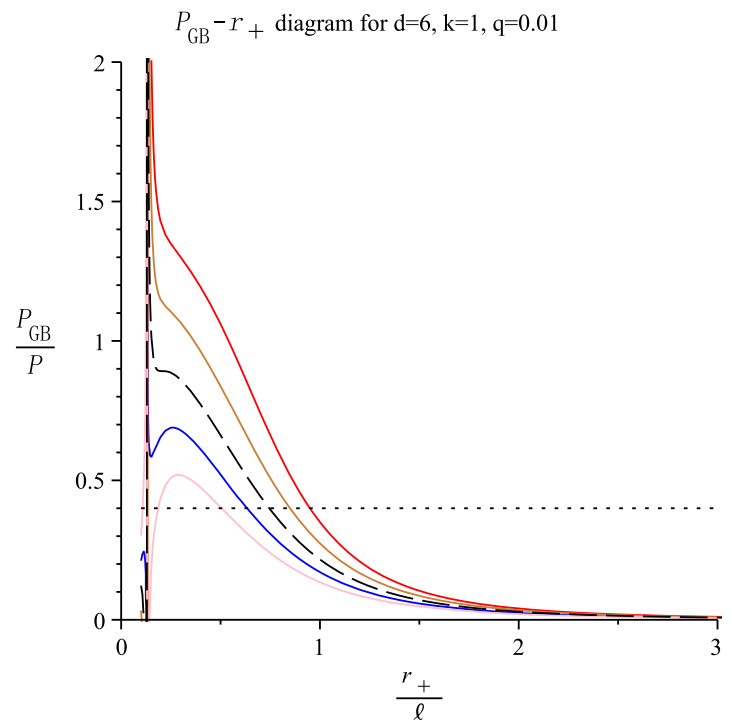

Fig. 4 The $P_{\mathrm{GB}}-r_{+}$(at arbitrary constant $\ell$ on the left) and $G-T$ (at $\ell=1$ on the right) diagrams for six dimensional static charged GB-AdS black holes with $q=0.01, k=1$. The isotherms are all near the lower critical temperature $T=T_{s}$ and the $G-T$ curves are all near the corre-

\subsubsection{Numerical results in $d=6$ dimensions}

To analyze the critical points at fixed charge $Q$ rather than fixed $\varrho$, we now work numerically in the case $d=6$. Assuming that the AdS radius $\ell$ is fixed and writing

$Q=q \ell^{3}, \quad r_{c}=\sigma_{c} \ell$,

where both $q$ and $\sigma_{c}$ are dimensionless, the critical point conditions can be reduced into the following equation for the critical radius parameter $\sigma_{c}$ :

$$
\begin{aligned}
& 175 q^{4}-48 \sigma_{c}^{6} q^{2}-4800 q^{2} \sigma_{c}^{8}+144 \sigma_{c}^{12}-2880 \sigma_{c}^{14} \\
& \quad+14400 \sigma_{c}^{16}=0 .
\end{aligned}
$$

Setting $q=0.01$, the critical radius parameter can be worked out numerically, giving rise to two different critical radii: a small critical radius $\sigma_{s} \approx 0.208$ with the corresponding critical temperature $T_{S} \approx \frac{0.350}{\ell}$ and critical thermodynamic pressure $P_{\mathrm{GB}}^{s} \approx \frac{0.355}{\ell^{2}}$ (i.e. $\frac{P_{\mathrm{GB}}^{s}}{P} \approx 0.892$ ) and a large critical radius $\sigma_{l} \approx 0.377$ with the corresponding temperature $T_{l} \approx$ $\frac{0.508}{\ell}$ and pressure $P_{\mathrm{GB}}^{l} \approx \frac{0.821}{\ell^{2}}$ (i.e. $\frac{P_{\mathrm{GB}}^{s}}{P} \approx 2.063$ ). The $P_{\mathrm{GB}}-r_{+}$and $G-T$ diagrams around each of these critical points are depicted in Figs. 4 and 5, respectively. One finds that the swallow tails appear either at $0.4 P<P_{\mathrm{GB}}<P_{\mathrm{GB}}^{s}$ (i.e. $0.4<\frac{P_{\mathrm{GB}}}{P}<0.892$ ), $T_{l}<T<T_{s}$ or at $P_{\mathrm{GB}}>P_{\mathrm{GB}}^{l}$ (i.e. $\left.\frac{P_{\mathrm{GB}}}{P}>2.063\right), T>T_{l}$ but not when $P_{\mathrm{GB}}^{s}<P_{\mathrm{GB}}<$ $P_{\mathrm{GB}}^{l}$, (i.e. $0.892<\frac{P_{\mathrm{GB}}}{P}<2.063$ ), $T_{s}<T<T_{l}$. Here $P_{G B}=0.4 P$ is precisely the lower bound for the pressure in six dimensions. When $T=T_{l}$, the pressure at the phase transition point happens to take a value at the lower bound.

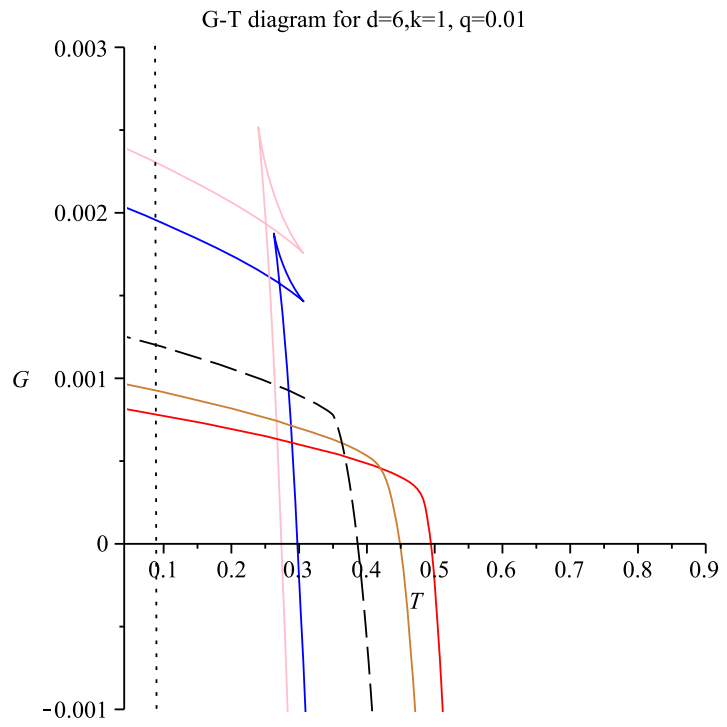

sponding critical pressure $P_{\mathrm{GB}}=P_{\mathrm{GB}}^{s}$. The dashed lines correspond to the critical curves and the dotted lines correspond to the lower bound for the pressure

The combination of Fig. 4 and Fig. 5 is given in Fig. 6, which gives the complete critical structure of the system at this particular value of electric charge.

\section{Conclusions}

Taking the (inverse of) GB coupling $\alpha$ as a new thermodynamic variable $P_{\mathrm{GB}}$, we revisited the thermodynamics for GB-AdS black holes and studied the associated critical behavior at fixed electric charge and bare cosmological constant. It is shown that for static neutral GB-AdS black holes, the corresponding critical point exists only for black holes with spherical topology (i.e. $k=+1$ ) in five dimensions, and the set of critical exponents are identical to those of the Van der Waals system. This is quite similar to the $P-V$ criticality associated with the cosmological constant at fixed GB coupling for the same black holes [22]. However, there is a crucial difference from the case of $P-V$ criticalities: in our case, the phase transition occurs only when the temperature is higher than the critical temperature, while the phase transition in $P-V$ criticalities occurs only when the temperature is lower than the critical temperature. The situation for static charged GB-AdS black holes is much more complicated, and it is shown that there can only be one critical point in five dimensions (for either $k=+1$ or $k=-1$ ) when the electric charge $Q$ obeys some appropriate bound. The corresponding critical exponents are also identical to those for the Van der Waals system. In higher dimensions, it is shown that there can be two critical points if the electric charge is 


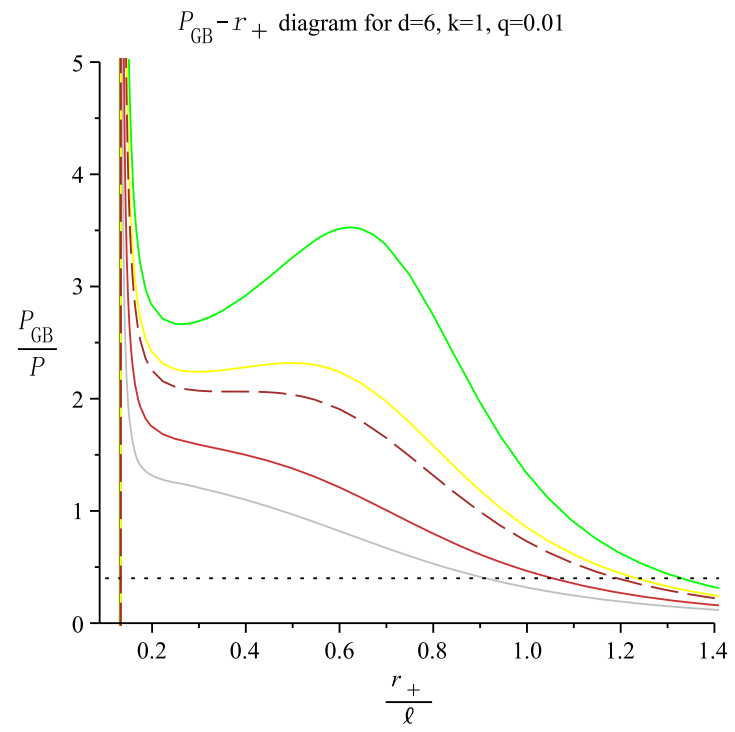

Fig. 5 The $P_{\mathrm{GB}}-r_{+}$(at arbitrary constant $\ell$ on the left) and $G-T$ (at $\ell=1$ on the right) diagrams for six dimensional static charged GB-AdS black holes with $q=0.01, k=1$. The isotherms are all near the higher critical temperature $T=T_{l}$ and the $G-T$ curves are all near the corre-

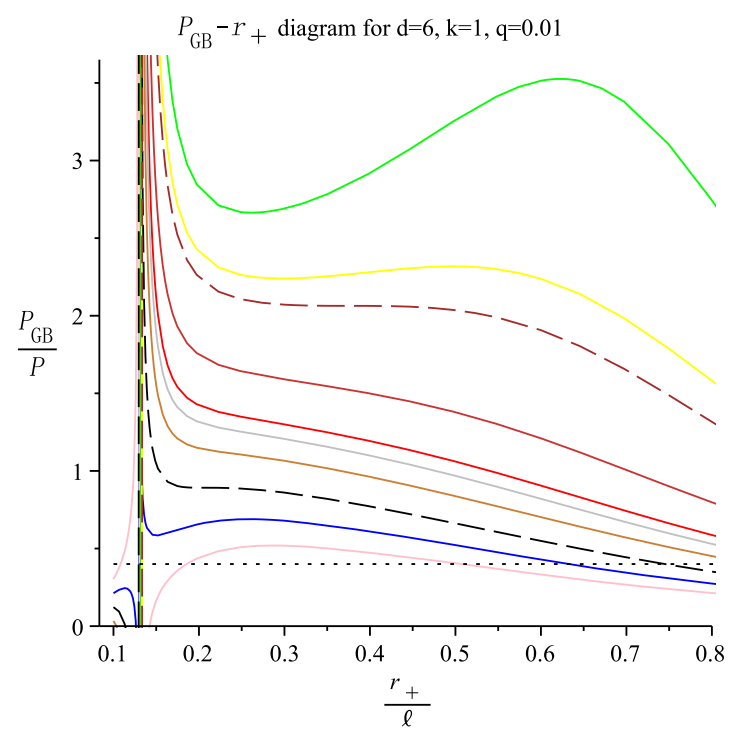

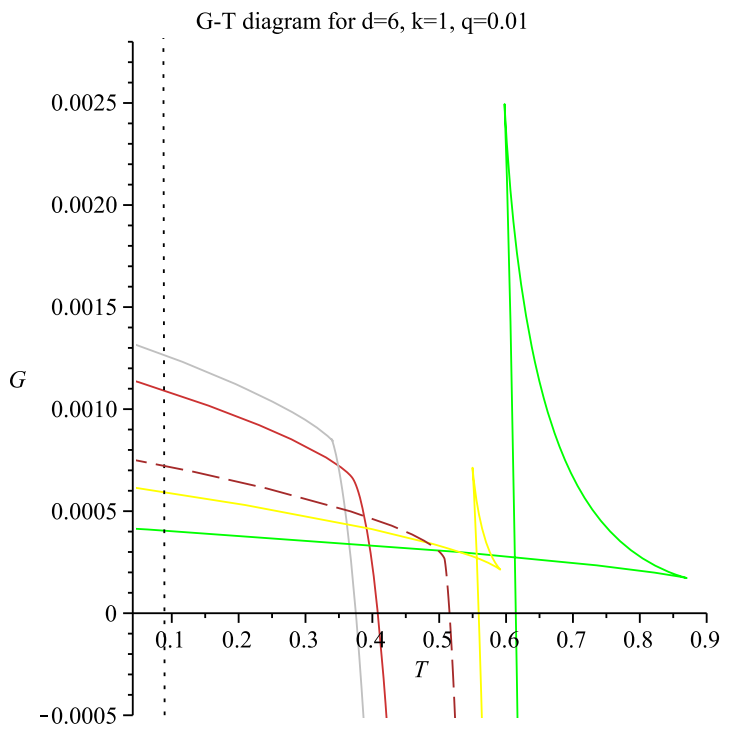

sponding critical pressure $P_{\mathrm{GB}}=P_{\mathrm{GB}}^{l}$. The dashed lines correspond to the critical curves and the dotted lines correspond to the lower bound for the pressure

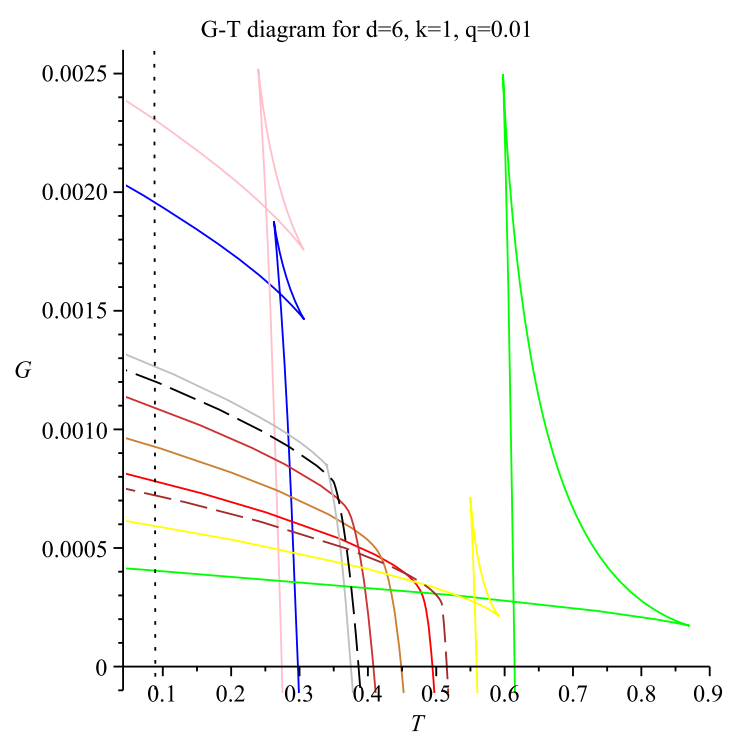

Fig. 6 The combination of Fig. 4 and Fig. 5. Note that the two critical isotherms on the left plots never cross each other; however, the corresponding $G-T$ curves do cross each other

taken to be proportional to the $(d-3)$ th power of the critical radius $\left(Q \propto r_{c}^{d-3}\right)$. A numerical study also shows that in six dimensions, there can be two different critical points at the same fixed electric charge, and the phase transitions can occur when the temperature is either lower than the lower critical temperature or higher than the higher critical temperature but not in between the two critical temperatures. This situation is not seen in earlier studies on $P-V$ criticalities for the same theory. Therefore, our study indicates that there are still much richer, unexpected structures in the thermodynamics of GB-AdS black holes.
Before closing, let us make some comments on the relation of our results and the $\alpha \rightarrow 0$ limit, in which the theory reduces to Einstein gravity. When $\alpha$ is vanishing, $P_{\mathrm{GB}}$ is infinite, and $V_{\mathrm{GB}}$ is identically zero, thus $P_{\mathrm{GB}}$ and $V_{\mathrm{GB}}$ lose their role as a pair of thermodynamic variables. This seems to invalidate our discussion at this particular limit. However, our results still stand because the criticalities described in this paper all appear at finite $P_{\mathrm{GB}}$ and so are invisible from the Einstein gravity limit.

It will be illuminating to look at the $G-P_{\mathrm{GB}}$ plots at fixed $T$ which are presented in Fig. 7. For each $G-P_{\mathrm{GB}}$ curve, 


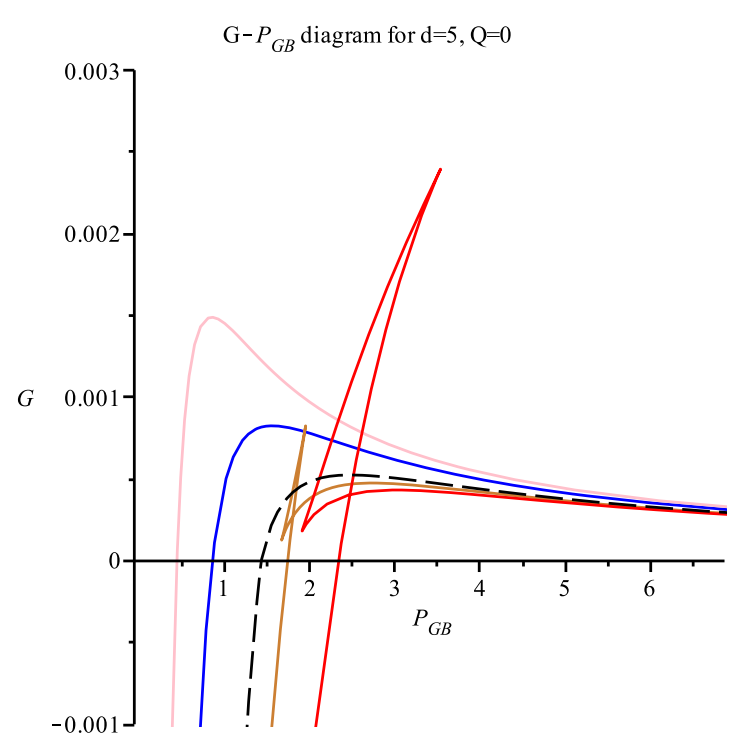

Fig. 7 The Gibbs free energy at fixed $T$ ( $G-P_{\mathrm{GB}}$ plots) for five dimensional static neutral GB-AdS black holes (with $k=1, \ell=1$ on the left) and for five dimensional static charged GB-AdS black holes (with $k=1, \ell=8$ and $Q=\frac{\sqrt{10}}{20} \ell^{2}$ on the right). The critical curves

the global minimum of the Gibbs free energy is " $\Gamma$ "-shaped, with the left steep branch corresponding to the stable large black hole phase (small $P_{\mathrm{GB}}$ and large $\alpha$ ) and the right gentle branch corresponding to the stable small black hole phase (large $P_{\mathrm{GB}}$ and small $\alpha$ ). One can also find the "swallow tail" corresponding to the first order phase transition, which is always located near the small $P_{\mathrm{GB}}$ end. That to say, at fixed $T$, one can never find a phase transition associated with the variable $P_{\mathrm{GB}}$ near the $\alpha \rightarrow 0$ limit ("Einstein gravity" black hole phase). The work presented in this paper indicates that the $\alpha \rightarrow 0$ limit is a metastable phase of the theory if the GB coupling $\alpha$ is to be considered as a thermodynamic variable, and the large $\alpha$ (small $P_{\mathrm{GB}}$ ) phase is thermodynamically preferred. On the other hand, the existence of a lower bound for $P_{\mathrm{GB}}$ ensures that $P_{\mathrm{GB}}$ will not go to zero in a thermodynamic process, so one does not need to worry about the possibility that $\alpha$ runs to infinity.

Open Access This article is distributed under the terms of the Creative Commons Attribution License which permits any use, distribution, and reproduction in any medium, provided the original author(s) and the source are credited. Funded by $\mathrm{SCOAP}^{3}$ / License Version CC BY 4.0.

\section{References}

1. J.M. Maldacena, The large $\mathrm{N}$ limit of superconformal field theories and supergravity. Adv. Theor. Math. Phys. 2, 231 (1998). arXiv:hep-th/9711200

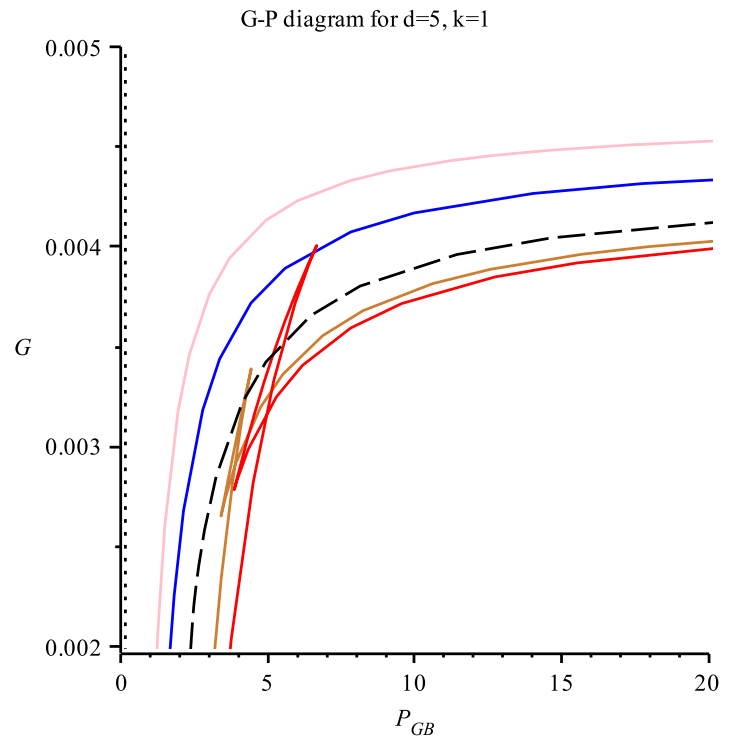

$T=T_{c}$ are depicted in dashed lines and the dotted lines correspond to the lower bound for the pressure. The temperature $T$ on the both plots increases from left to right, and the "swallow tail" behavior appears only when $T>T_{C}$, which corresponds to a first order phase transition

2. S.S. Gubser, I.R. Klebanov, A.M. Polyakov, Gauge theory correlators from noncritical string theory. Phys. Lett. B 428, 105 (1998). arXiv:hep-th/9802109

3. E. Witten, Anti-de Sitter space and holography. Adv. Theor. Math. Phys. 2, 253 (1998). arXiv:hep-th/9802150

4. J.D. Brown, M. Henneaux, Central charges in the canonical realization of asymptotic symmetries: an example from three-dimensional gravity. Commun. Math. Phys. 104, 207 (1986)

5. S.W. Hawking, D.N. Page, Thermodynamics of black holes in antiDe Sitter space. Commun. Math. Phys. 87, 577 (1983)

6. A. Chamblin, R. Emparan, C.V. Johnson, R.C. Myers, Charged AdS black holes and catastrophic holography. Phys. Rev. D 60, 064018 (1999). arXiv:hep-th/9902170

7. A. Chamblin, R. Emparan, C.V. Johnson, R.C. Myers, Holography, thermodynamics and fluctuations of charged AdS black holes. Phys. Rev. D 60, 104026 (1999). arXiv:hep-th/9904197

8. Y.-D. Tsai, X.N. Wu, Y. Yang, Phase structure of Kerr-AdS black hole. Phys. Rev. D 85, 044005 (2012). arXiv:1104.0502

9. C. Niu, Y. Tian, X.-N. Wu, Critical phenomena and thermodynamic geometry of RN-AdS black holes. Phys. Rev. D 85, 024017 (2012). arXiv: 1104.3066

10. R. Banerjee, D. Roychowdhury, Critical phenomena in Born-Infeld AdS black holes. Phys. Rev. D 58, 044040 (2012). arXiv:1111.0147

11. R. Banerjee, D. Roychowdhury, Critical behavior of Born Infeld AdS black holes in higher dimensions. Phys. Rev. D 85, 104043 (2012). arXiv: 1203.0118

12. A. Lala, Critical phenomena in higher curvature charged AdS black holes. Adv. High Energy Phys. 2013, 918490 (2013). arXiv: 1205.6121

13. S.-W. Wei, Y.-X. Liu, Critical phenomena and thermodynamic geometry of charged GB AdS black holes. Phys. Rev. D 87(4), 044014 (2013). arXiv: 1209.1707

14. M.M. Caldarelli, G. Cognola, D. Klemm, Thermodynamics of Kerr-Newman-AdS black holes and conformal field theories. Class. Quant. Grav. 17, 399 (2000). arXiv:hep-th/9908022

15. D. Kastor, S. Ray, J. Traschen, Enthalpy and the mechanics of AdS black holes. Class. Quant. Grav. 26, 195011 (2009). arXiv:0904.2765 
16. B.P. Dolan, The cosmological constant and the black hole equation of state. Class. Quant. Grav. 28, 125020 (2011). arXiv:1008.5023

17. B.P. Dolan, Pressure and volume in the first law of black hole thermodynamics. Class. Quant. Grav. 28, 235017 (2011). arXiv: 1106.6260

18. B.P. Dolan, Compressibility of rotating black holes. Phys. Rev. D 84, 127503 (2011). arXiv:1109.0198

19. B.P. Dolan, The compressibility of rotating black holes in D-dimensions. Class. Quant. Grav. 31, 035022 (2014). arXiv: 1308.5403

20. M. Cvetic, G.W. Gibbons, D. Kubiznak, C.N. Pope, Black hole enthalpy and an entropy inequality for the thermodynamic volume. Phys. Rev. D 84, 024037 (2011). arXiv:1012.2888

21. H. Lu, Y. Pang, C.N. Pope, J.F. Vazquez-Poritz, AdS and Lifshitz black holes in conformal and Einstein-Weyl gravities. Phys. Rev. D 86, 044011 (2012). arXiv: 1204.1062

22. R.-G. Cai, L.-M. Cao, L. Li, R.-Q. Yang, P-V criticality in the extended phase space of GB black holes in AdS space. JHEP 1309, 005 (2013). arXiv: 1306.6233

23. D. Kubiznak, R.B. Mann, P-V criticality of charged AdS black holes. JHEP 1207, 033 (2012). arXiv: 1205.0559

24. S. Gunasekaran, R.B. Mann, D. Kubiznak, Extended phase space thermodynamics for charged and rotating black holes and Born-Infeld vacuum polarization. JHEP 1211, 110 (2012). arXiv: 1208.6251

25. B.P. Dolan, Where is the PdV term in the fist law of black hole thermodynamics? arXiv: 1209.1272

26. A. Belhaj, M. Chabab, H. El Moumni, M.B. Sedra, On thermodynamics of AdS black holes in arbitrary dimensions. Chin. Phys. Lett. 29, 100401 (2012). arXiv:1210.4617

27. S.H. Hendi, M.H. Vahidinia, P-V criticality of higher dimensional black holes with nonlinear source. Phys. Rev. D 88(8), 084045 (2013). arXiv:1212.6128

28. S. Chen, X. Liu, C. Liu, J. Jing, $P-V$ criticality of AdS black hole in $f(R)$ gravity. Chin. Phys. Lett. 30, 060401 (2013). arXiv: 1301.3234

29. K. Hristov, C. Toldo, S. Vandoren, Phase transitions of magnetic AdS4 black holes with scalar hair. Phys. Rev. D 88, 026019 (2013). arXiv: 1304.5187

30. E. Spallucci, A. Smailagic, Maxwell's equal area law for charged anti-deSitter black holes. Phys. Lett. B 723, 436 (2013). arXiv: 1305.3379

31. R. Zhao, H.-H. Zhao, M.-S. Ma, L.-C. Zhang, On the critical phenomena and thermodynamics of charged topological dilaton AdS black holes. Eur. Phys. J. C 73, 2645 (2013). arXiv:1305.3725

32. A. Belhaj, M. Chabab, H.E. Moumni, M.B. Sedra, Critical behaviors of 3D black holes with a scalar hair. arXiv:1306.2518

33. M.B.J. Poshteh, B. Mirza, Z. Sherkatghanad, Phase transition, critical behavior, and critical exponents of Myers-Perry black holes. Phys. Rev. D 88, 024005 (2013). arXiv: 1306.4516
34. A. Belhaj, M. Chabab, H.E. Moumni, L. Medari, M.B. Sedra, The thermodynamical behaviors of Kerr-Newman AdS black holes. Chin. Phys. Lett. 30, 090402 (2013). arXiv:1307.7421

35. N. Altamirano, D. Kubiznak, R.B. Mann, Z. Sherkatghanad, KerrAdS analogue of tricritical point and solid/liquid/gas phase transition. Class. Quant. Grav. 31, 042001 (2014). arXiv:1308.2672

36. B.P. Dolan, D. Kastor, D. Kubiznak, R.B. Mann, J. Traschen, Thermodynamic volumes and isoperimetric inequalities for de Sitter black holes. Phys. Rev. D 87, 104017 (2013). arXiv:1301.5926

37. W. Ballik, K. Lake, The vector volume and black holes. Phys. Rev. D 88(10), 104038 (2013). arXiv:1310.1935

38. G.W. Gibbons, R. Kallosh, B. Kol, Moduli, scalar charges, and the first law of black hole thermodynamics. Phys. Rev. Lett. 77, 4992 (1996). arXiv:hep-th/9607108

39. J.D.E. Creighton, R.B. Mann, Quasilocal thermodynamics of dilaton gravity coupled to gauge fields. Phys. Rev. D 52, 4569 (1995). arXiv:gr-qc/9505007

40. D.A. Rasheed, Nonlinear electrodynamics: zeroth and first laws of black hole mechanics. arXiv:hep-th/9702087

41. N. Breton, Smarr's formula for black holes with non-linear electrodynamics. Gen. Rel. Grav. 37, 643 (2005). arXiv:gr-qc/0405116

42. W. Yi-Huan, Energy and first law of thermodynamics for BornInfeld AdS black hole. Chin. Phys. B 19, 090404 (2010)

43. D.G. Boulware, S. Deser, String generated gravity models. Phys. Rev. Lett. 55, 2656 (1985)

44. R.-G. Cai, GB black holes in AdS spaces. Phys. Rev. D 65, 084014 (2002). arXiv:hep-th/0109133

45. M.H. Dehghani, Asymptotically (anti)-de Sitter solutions in GB gravity without a cosmological constant. Phys. Rev. D 70, 064019 (2004). arXiv:hep-th/0405206

46. W. Xu, K. Meng, L. Zhao, Accelerating vacua in GB gravity. Commun. Theor. Phys. 58, 59 (2012). arXiv:1110.5769

47. L. Zhao, K. Meng, GB as effective cosmological constant. Commun. Theor. Phys. 57, 607 (2012). arXiv:1109.6748

48. D. Kastor, S. Ray, J. Traschen, Smarr formula and an extended first law for lovelock gravity. Class. Quant. Grav. 27, 235014 (2010). arXiv: 1005.5053

49. D.L. Wiltshire, Spherically symmetric solutions of EinsteinMaxwell theory with a GB term. Phys. Lett. B 169, 36 (1986)

50. M. Cvetic, S. 'i. Nojiri, S.D. Odintsov, Black hole thermodynamics and negative entropy in de Sitter and anti-de Sitter Einstein-GB gravity. Nucl. Phys. B 628, 295 (2002). arXiv:hep-th/0112045

51. T. Clunan, S.F. Ross, D.J. Smith, On GB black hole entropy. Class. Quant. Grav. 21, 3447 (2004). arXiv:gr-qc/0402044

52. R.E. Reichl, A Modern Course in Statistical Physics (University of Texas Press, Austin, 1980) 